\title{
Matérista
}

Revista Matéria, v. 14, n. 4, pp. 1134 - 1145, 2009

ISSN 1517-7076

http://www.materia.coppe.ufrj.br/sarra/artigos/artigo11119

\section{Estudo de relaxações dielétricas e dinâmico-mecânicas da borracha natural por meio das técnicas de DMTA E TSC}

\author{
GOMES $^{\mathrm{I}}$, D.M.; KOWALSKI ${ }^{\mathrm{II}}$, E. L.; MUNARO ${ }^{\mathrm{II}}$, M.; ROBERT ${ }^{\mathrm{II}}$, E.R. \\ I Departamento de Eletromecânica, Instituto de Tecnologia para o Desenvolvimento - LACTEC, \\ Universidade Federal do Paraná, CP: 19067, 81531-980, Curitiba - PR.. \\ e-mail: daisysamelo@yahoo.com.br \\ II Departamento de Eletromecânica, Instituto de Tecnologia para o Desenvolvimento - LACTEC, \\ Universidade Federal do Paraná, CP: 19067, 81531-980, Curitiba - PR. \\ e-mail: edemir@lactec.org.br, marilda@lactec.org.br, rene@lactec.org.br
}

\section{RESUMO}

A borracha natural (BN) é amplamente utilizada para aplicações em isolamento elétrico. No setor elétrico, BN é usada principalmente na fabricação de equipamentos de segurança como luvas, mangas e lençóis isolantes e ferramentas aplicadas em serviços com a rede energizada. Este trabalho faz parte de uma pesquisa sobre propriedades elétricas e mecânicas da BN. Foram obtidos resultados por meio das técnicas de corrente termicamente estimulada - TSC (thermally stimulated current), uma importante técnica de caracterização de armazenamento de carga em BN, análise termodinâmico-mecânica - DMTA (dynamic mechanical thermal analysis). Duas formulações foram estudadas, uma contendo enxofre como agente de vulcanização (cura) e a outra, peróxido de dicumila. A reorientação de dipolos elétricos detectados por TSC para as amostras de borracha natural, novas e envelhecidas de ambas as formulações, estão relacionados aos movimentos de segmentos, grupos laterais, e partes de terminações de cadeias, detectados por DMTA. Além disso, a degradação das amostras de borracha natural mostrou-se divida em dois estágios: no primeiro, a reação de reticulação é predominante e, no segundo, predominam a cisão de cadeias e a reação de oxidação de terminações, ramificações e pontos de cisão.

Palavras-chaves: TSC, borracha natural, DMTA.

\section{Study of dielectric and dynamic mechanical relaxations of the natural rubber through DMTA and TSC spectroscopy}

\section{ABSTRACT}

Natural Rubber (NR) is widely used in applications where electric insulation is required. In power systems utilities, NR is used mainly to manufacture live line security equipments - rubber insulating gloves, sleeves, matting and electric isolation tools. This work is part of a research about the electric and mechanicals properties of NR. Results from thermally stimulated current - TSC spectroscopy, an important characterization method of charge storage in NR, and dynamic mechanical thermal analysis - DMTA are presented. Two compositions of NR have been studied, one contain sulfur as vulcanization (cure) agent, and the other with dicumile peroxide. The redirection of electric dipoles detected by TSC for new and aging natural rubber samples, of the two formulations are related to movements of segments, side groups, and parts of terminations of chains, detected by DMTA. Furthermore, the degradation of natural rubber samples proved to be divided into two stages: firstly, the cross-linking reaction is predominant and in the second, the predominant chain scission and oxidation reaction of endings, branches and scission points.

Keywords: TSC, natural rubber, DMTA.

\section{INTRODUÇÃO}

A borracha natural é amplamente aplicada no setor elétrico como, por exemplo, em ferramentas para eletricistas como luvas, mangas e lençóis isolantes utilizados em serviços com a rede energizada [1-3]. A forma de armazenamento, o tempo de uso e a exposição ao meio ambiente provocam a degradação da 
borracha natural, alterando as suas propriedades isolantes, bem como suas propriedades mecânicas. Sendo assim, tais fatores podem colocar em risco a vida do trabalhador de linha viva. Se mal armazenado, por exemplo, o material pode sofrer degradação pela ação térmica, ozônio e radiação UV. Para produzir ferramentas e equipamentos de boa qualidade é necessário para sua formulação conhecer características com relação à sua aplicação, processos de degradação e a compatibilidade desejada com outros equipamentos. Estudos sobre as propriedades elétricas e mecânicas e os efeitos causados pelo envelhecimento térmico são de grande importância para as aplicações da borracha natural [1-ㅡ] .

As propriedades elétricas relacionadas ao fenômeno de polarização elétrica por dipolos ou cargas espaciais na borracha natural podem ser estudadas através da espectroscopia de Corrente Termicamente Estimulada, TSC [늠].

As propriedades mecânicas podem ser estudadas em termos da viscoelasticidade do elastômero [ㅁ10], pela técnica de análise termodinâmico-mecânica - DMTA [11-14], por medidas de módulo de elasticidade e tangente delta.

Para obter-se borracha com melhores propriedades dentro da aplicação desejada, procede-se adição de cargas reforçantes e aditivos que, respectivamente, melhoram suas propriedades e minimizam os efeitos da degradação, agentes vulcanizadores, com ou sem aceleradores, tornando a estrutura da borracha natural bastante heterogênea. Neste trabalho são analizadas medidas de TSC e DMTA para duas diferentes formulações químicas de borracha natural em tempos diferentes de envelhecimento. Uma das amostras contém enxofre como agente de vulcanização (cura), e na outra, o processo de cura é feito por peróxido de dicumila.

\section{MATERIAIS E MÉTODOS}

\subsection{Formulações}

As amostras dos compostos de borracha natural foram feitas em moinho padrão, pela Empresa Polirubber, seguindo a seqüência abaixo:

1 plastificação ou mastigação da borracha natural por 5 minutos na temperatura média de $50{ }^{\circ} \mathrm{C}$ e cilindros com abertura de 0,5 mm rotação 25 x 34 rpm e fricção 1:1,4;

2 adição de anti-oxidante TMQ (2,2,4 - trimetil-1,2-dihidroquinolina);

3 adição de carga mineral-negro de fumo N-762;

Tabela 1: Formulação da borracha natural curada por enxofre.

\begin{tabular}{c|c}
\hline $\begin{array}{c}\text { Componentes/Tipo de } \\
\text { Cura }\end{array}$ & A5/Enxofre \\
\hline borracha SMR-L & $100 \mathrm{phr}$ \\
\hline anti-oxidante TMQ & 2 \\
\hline óxido de zinco & 5 \\
\hline ácido esteárico & 1 \\
\hline Struktol WB-212 & 1 \\
\hline negro de fumo N-762 & 5 \\
\hline Flexpar 848 & 5 \\
\hline enxofre & 1 \\
\hline aceleradores TMTD & 1 \\
\hline aceleradores MBT & 0,5 \\
\hline
\end{tabular}


Tabela 2: Formulação da borracha natural curada por peróxido de dicumila.

\begin{tabular}{c|c}
\hline Composto/Tipo de Cura & A5/Peróxido de Dicumila \\
\hline borracha SMR-L & $100 \mathrm{phr}$ \\
\hline anti-oxidante TMQ & 2 \\
\hline óxido de zinco & 5 \\
\hline Struktol WB-212 & 1 \\
\hline negro de fumo N-762 & 5 \\
\hline Flexpar 848 & 5 \\
\hline peróxido de dicumila & 5 \\
\hline
\end{tabular}

cura da borracha natural:

a. Borracha A5E - 5 phr de negro de fumo e sistema de cura via enxofre com adição de óxido de zinco, ácido esteárico e aceleradores TMTD (Dissulfeto de tetrametil tiuran) e MBT (2Mercaptobenzotiazol) Tabela 1;

b. Borracha A5P - 5 phr de negro de fumo e sistema de cura via peróxido de dicumila. Neste caso, retira-se o ácido esteárico da formulação, pois o mesmo pode retardar o processo de entrecruzamento das cadeias do polímero (Tabela 2);

5 homogeneização, através de 10 passagens da mistura pelo cilindro.

As duas formulações são mostradas nas Tabelas 1 e 2, com as frações dos componentes em partes por cem partes de borracha natural (phr) .

\subsection{Preparação das amostras}

Os filmes de borracha natural com espessura média de 0,7 mm, e 60x60 $\mathrm{mm}^{2}$ de área foram obtidos pelo processo de termo-prensagem, onde foram conformados e vulcanizados em termo-prensa hidráulica, com capacidade de 60 toneladas métricas. A temperatura e o tempo de cura foram respectivamente $150{ }^{\circ} \mathrm{C} \mathrm{e}$ 5 minutos, sob força constante de intensidade $10 \mathrm{kN}$.

\subsection{Envelhecimento das amostras}

As amostras de borracha natural foram envelhecidas termicamente à temperatura de $70^{\circ} \mathrm{C}$. $\mathrm{O}$ envelhecimento térmico para as duas formulações foi feito em estufa marca Fanem com circulação mecânica modelo $320 \mathrm{SE}$. As siglas utilizadas para identificar as amostras segundo o tempo de envelhecimento são mostradas na Tabela 3.

Tabela 3: Envelhecimento da borracha natural curada por enxofre - A5E.

\begin{tabular}{c|c|c}
\hline $\begin{array}{c}\text { Tempo de } \\
\text { Envelhecimento }\end{array}$ & $\begin{array}{c}\text { Composto/Cura por } \\
\text { Enxofre }\end{array}$ & $\begin{array}{c}\text { Composto/Cura com } \\
\text { Peróxido de Dicumila }\end{array}$ \\
\hline $\begin{array}{c}\text { Amostras não } \\
\text { envelhecidas }\end{array}$ & A5E & A5P \\
\hline 360 horas (2 semanas) & A5E360 & A5P360 \\
\hline 720 horas (4 semanas) & A5E720 & A5P720 \\
\hline 1080 horas (6 semanas) & A5E1080 & A5P1080 \\
\hline
\end{tabular}




\section{DMTA}

Para análise termodinâmico-mecânica - DMTA foi utilizado o analisador dinâmico-mecânico DMA da marca Netzsch, modelo 242. A taxa de temperatura e a faixa de varredura foram $3^{\circ} \mathrm{C} / \mathrm{min}$ e de $130^{\circ} \mathrm{C}$ a $70^{\circ} \mathrm{C}$, respectivamente, com resfriamento das amostras sob atmosfera de nitrogênio. As frequiências adotadas para a força variável aplicada foram de $1 \mathrm{~Hz}, 3,3 \mathrm{~Hz}$ e $5 \mathrm{~Hz}$.

\section{$2.5 \quad$ TSC}

Primeiramente, foi necessária remoção de impurezas das superfícies dos eletrodos com acetona e das amostras com álcool isopropílico. Logo após, a amostra foi inserida entre dois eletrodos de cobre dentro da câmara de criogenia. Os eletrodos, em forma de discos, possuiam diâmetros de $45 \mathrm{~mm}$ e $55 \mathrm{~mm}$ ligados a uma fonte DC. A pressão obtida dentro da câmara de criogenia foi em torno de $10^{-5}$ Torr.

Com a câmara operando na faixa de pressão desejada, a amostra foi submetida a curto-circuito, por 30 minutos e, logo após, aquecida à temperatura de $100^{\circ} \mathrm{C}$, quando foi aplicada a tensão de $500 \mathrm{~V}$ DC. Com o campo elétrico aplicado, a amostra foi até $-196^{\circ} \mathrm{C}$, com a intenção de "congelar" os dipolos permanentes e induzidos orientados no volume do material. Mantendo-se a temperatura constante, o campo foi removido, e a amostra novamente foi submetida a curto-circuito para que possíveis correntes superficiais fossem eliminadas. Um eletrômetro foi conectado em série ao circuito para a leitura de corrente na amostra. Aumentando-se a temperatura a uma taxa de $5^{\circ} \mathrm{C} / \mathrm{min}$, foi possível a leitura de corrente elétrica de despolarização em função da temperatura. Estes procedimentos foram repetidos para as duas formulações com diferentes tempos de envelhecimento térmico, para a faixa de temperatura entre $-100^{\circ} \mathrm{C}$ e $0^{\circ} \mathrm{C}$.

\section{RESULTADOS E DISCUSSÕES}

\subsection{DMTA versus TSC em função do envelhecimento térmico}

O comportamento dinâmico-mecânico da borracha natural nas duas formulações, A5E e A5P foi estudado por meio das medidas do módulo elástico, da tangente de perdas, tan $\delta$, e alongamento em função da temperatura, para os tempos de envelhecimento mostrados nas tabelas 3 e 4 .

Os termogramas de corrente elétrica de despolarização $\mathrm{i}(\mathrm{T})$ versus temperatura $\mathrm{T}$ das medidas de TSC feitas para as amostras A5E (cura via enxofre) e A5P (cura via peróxido), foram obtidos entre $-100{ }^{\circ} \mathrm{C}$ e $0{ }^{\circ} \mathrm{C}$, a fim de compará-los com os resultados de DMTA, que ocorrem nessa região de temperatura.

Nos resultados obtidos por TSC, foi percebida a presença de um pico de corrente elétrica de despolarização, tanto para as amostras A5E como A5P. Este comportamento variou em função do envelhecimento térmico das amostras, como pode ser observado nas Figuras 2, 4, 6 e 8.

Os picos de corrente elétrica de despolarização estão relacionados às reorientações de dipolos permanentes formados por átomos, moléculas e segmentos de cadeias, resultando em movimentos mais localizados. Esta mobilidade restrita pode estar relacionada à transição vítrea do material, caracterizada pela passagem do estado vítreo para um estado "maleável”, sem ocorrência de uma mudança estrutural, isto é, um rearranjo de segmentos das cadeias, dependente do volume livre do material, em temperaturas mais baixas no caso da borracha natural. A questão, no entanto, é avaliar como os movimentos moleculares, de ramificações, segmentos ou terminações de cadeias estão relacionados com os picos de perdas detectados no DMTA e TSC, e avaliar a influência da formulação e do envelhecimento térmico sobre as propriedades da borracha natural [4-6].

Para as amostras não envelhecidas, os resultados mostrados na Figura 1 mostram um pico acentuado nos valores de tan $\delta$, em $-84^{\circ} \mathrm{C}$ para $\mathrm{A} 5 \mathrm{E}, \mathrm{e}-88^{\circ} \mathrm{C}$ para A5P. É possível ver também a presença de um menor pico sobreposto em torno de $-48^{\circ} \mathrm{C}$ e $-53^{\circ} \mathrm{C}$, para A5E e A5P, respectivamente. O primeiro pico mais intenso, é atribuído aos movimentos de curto alcance na estrutura do polímero [15], para ambas as formulações, bem como relaxações de grupos laterais ou ramificações [16] (ácidos carboxílicos, ésteres, metila e hidrogênio), pequenos segmentos de cadeias e/ou terminações de cadeias, bem como partes das proteínas e lipídios existentes nessas terminações [8]. O segundo pico pode ser atribuído aos movimentos de segmentos mais longos na estrutura. Estes picos estão acompanhados de uma queda abrupta no módulo elástico de ambas as composições, o que corresponde de acordo com a literatura [11,12], à transição vítrea borrachosa, $T_{\mathrm{g}}$, da borracha natural. $\mathrm{E}$ ainda é possível detectar a temperatura de mudança no coeficiente da curva de alongamento (onset), detectado pelo analisador dinâmico-mecânico, em torno de $-68^{\circ} \mathrm{C} \mathrm{e}-70^{\circ} \mathrm{C}$, para A5E e A5P respectivamente. Este valor de temperatura detectado pela mudança na alongamento das amostras pode servir como parâmetro da transição vítrea da borracha natural, por ser o mais relatado pela literatura, inclusive detectado por outras técnicas [12-14,17] 

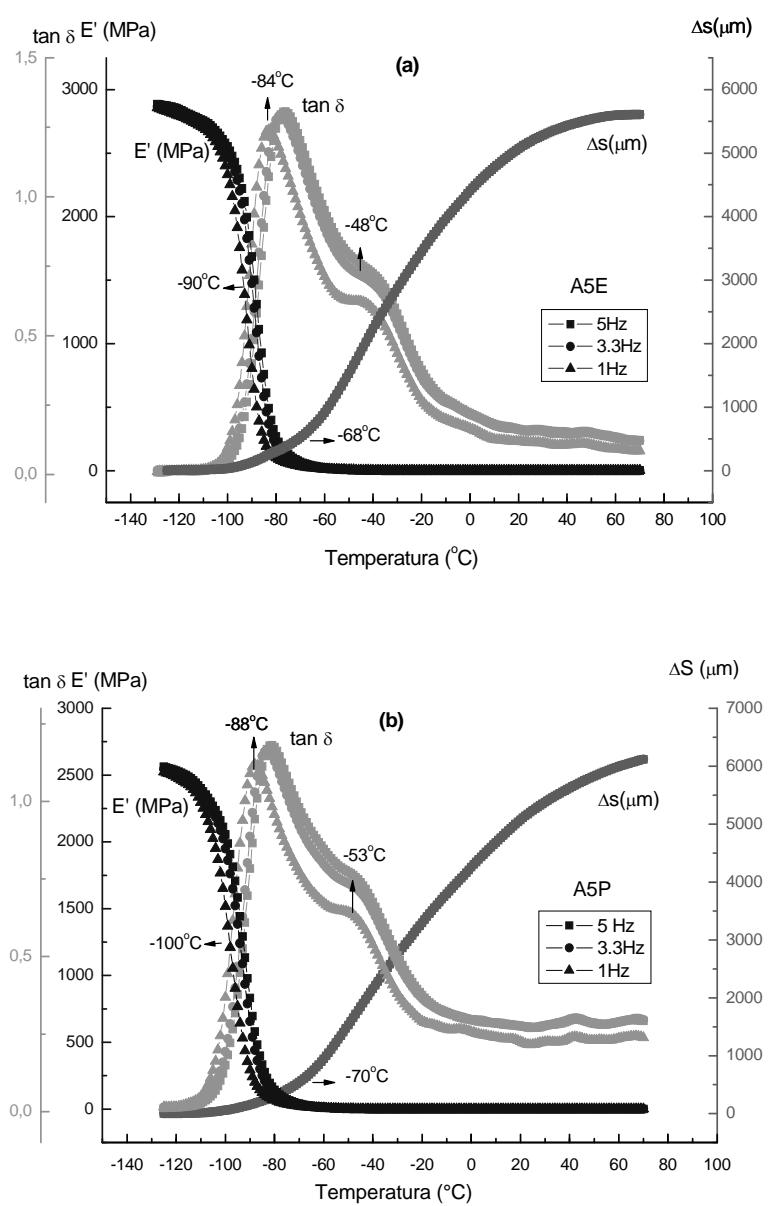

Figura 1: Medidas de Tan $\delta$, módulo elástico E’ e alongamento $\Delta$ s, em função da temperatura, obtidas pela técnica de DMTA, para as amostras (a) A5E e (b) A5P, não envelhecidas.

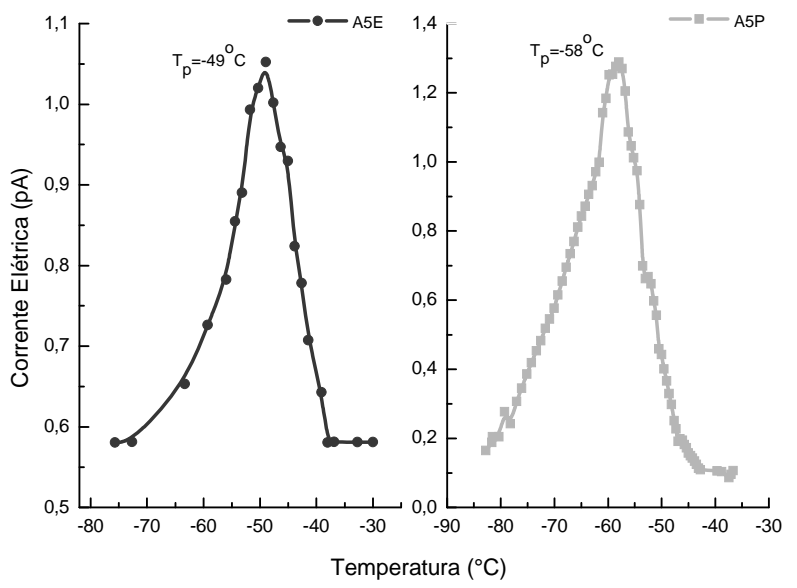

Figura 2: Medidas de TSC para amostras A5E e A5P não envelhecidas.

A Figura 2 apresenta os termogramas de TSC para ambas as formulações de BN não envelhecidas. As medidas de TSC para as formulações A5E e A5P não envelhecidas acompanham a tendência detectada no DMTA que mostra pico de relaxação em temperatura menor para a borracha natural curada com peróxido de dicumila. Isto sugere uma menor energia de ativação necessária para reorientar os dipolos na estrutura da amostra A5P, se comparada a A5E, concordando com a hipótese de que ocorre maior entrecruzamento na 
borracha vulcanizada por enxofre, deixando as estruturas polares mais presas. Sugere-se ainda que ocorra maior polarização das moléculas por interação dipolar, já que estão mais próximas umas das outras. Essa análise poderia ser feita, levando em consideração as intensidades de corrente de despolarização, com o envelhecimento, porém não entram no escopo deste trabalho. O interesse maior, neste momento está voltado aos mecanismos que provocam a corrente de despolarização e a relação com mecanismos de relaxação mecânica com envelhecimento.
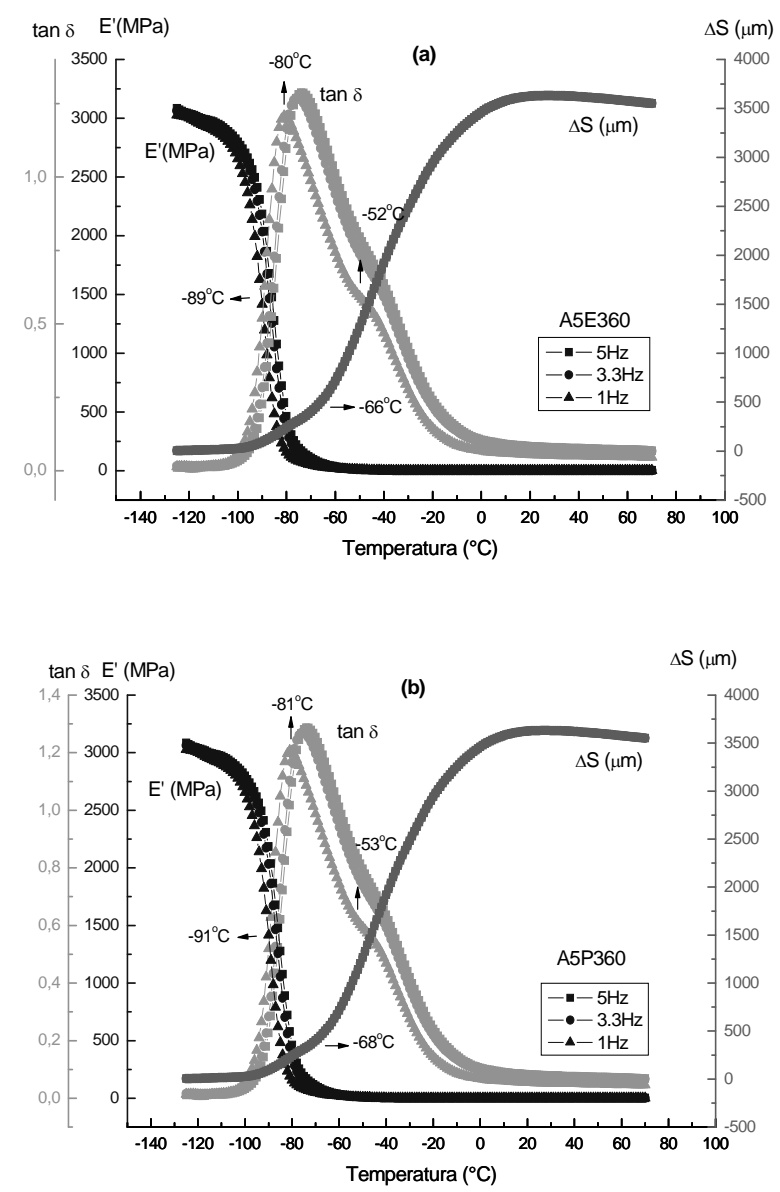

Figura 3: Medidas de Tan $\delta$, módulo elástico E’ e alongamento $\Delta$ s, em função da temperatura, obtidas pela técnica de DMTA, para as amostras (a) A5E360 e (b) A5P360, envelhecidas por 360 horas (2 semanas).

A Figura 3 mostra as medidas de DMTA para as amostras envelhecidas termicamente a $70^{\circ} \mathrm{C}$ por 360h (2 semanas). Observa-se na que as temperaturas de onset dinâmico-mecânicas tendem a aumentar ligeiramente para ambas as formulações, A5E e A5P, à princípio sem diferença significativa deste comportamento entre as duas, o que pode ser melhor visualizado nos valores de energia de ativação, ainda nesta seção. A elevação nos valores de temperatura pode ser atribuído ao efeito de pós cura, que se trata do aumento no número de entrecruzamentos das cadeias, durante o envelhecimento térmico, relacionado ao processo de degradação da borracha natural, onde predomina a reação reticulação perante à degradação oxidativa. 


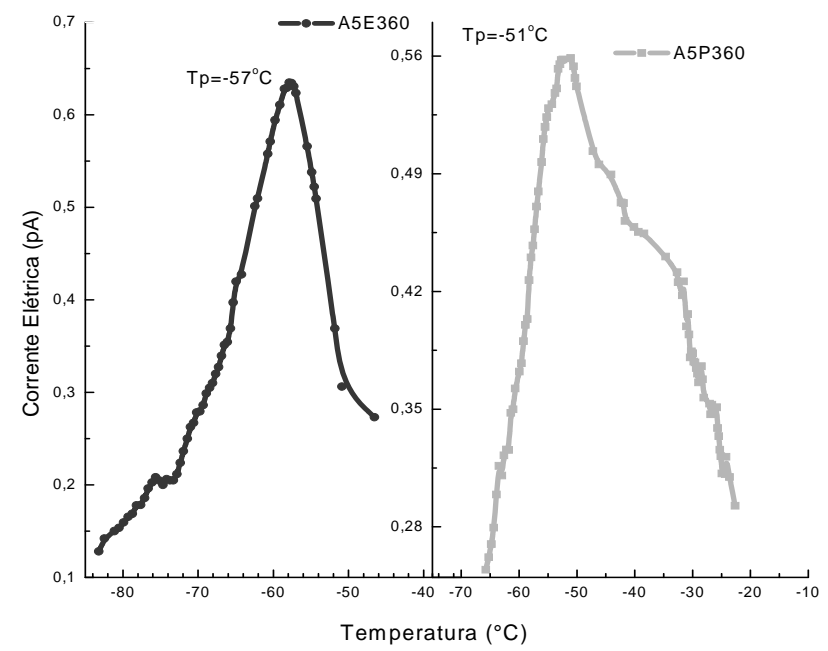

Figura 4: Medidas de TSC para amostras A5E360 e A5P360, envelhecidas por 360 horas (2 semanas).

As medidas de TSC, para 360 horas de envelhecimento, conforme Figura 4, também acompanham a tendência detectada no DMTA, para este envelhecimento, onde verifica-se que as temperaturas de relaxação tanto mecânica quanto dielétrica aumentam em função do estágio de reticulação referente ao efeito de degradação de ambas as estruturas
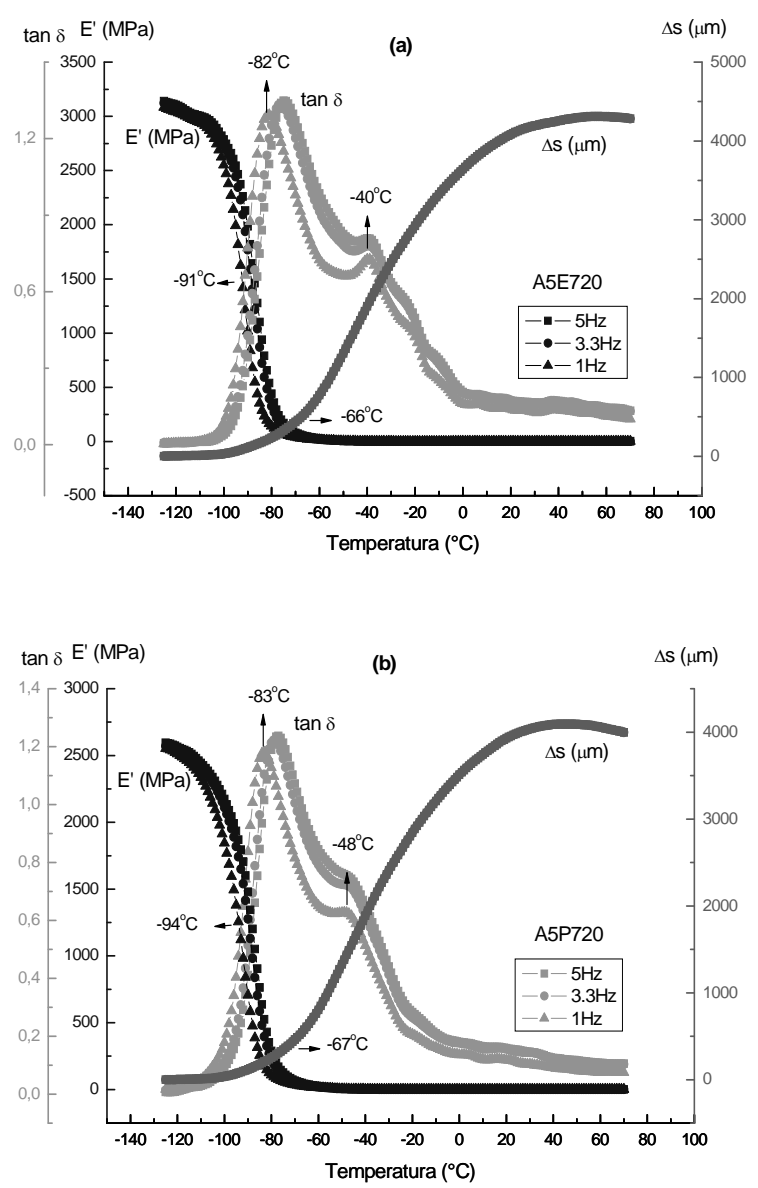

Figura 5: Medidas de Tan $\delta$, módulo elástico E’ e alongamento $\Delta$ s, em função da temperatura, obtidas pela técnica de DMTA, para as amostras (a) A5E720 e (b) A5P720, envelhecidas por 720 horas (4 semanas). 
As Figuras 5 e 6 mostram os resultados das medidas de DMTA e TSC, respectivamente, para as amostras envelhecidas termicamente a $70^{\circ} \mathrm{C}$ por $720 \mathrm{~h}$ (4 semanas). Observou-se que as temperaturas de relaxação de TSC tendem a valores maiores, acompanhando a tendência dos mecanismos de relaxação dinâmico-mecânicos detectados pelo DMTA, o que é atribuído também ao efeito de pós cura, que como já comentado, é a reticulação continuada da estrutura da borracha natural, para ambas as formulações, A5E e A5P.

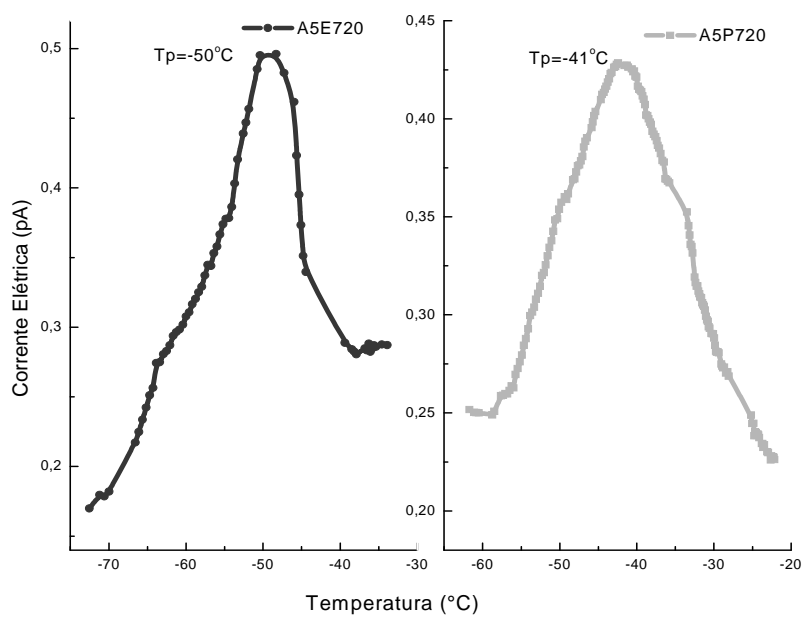

Figura 6: Medidas de TSC para amostras A5E720 e A5P720, envelhecidas por 720 horas (4 semanas).
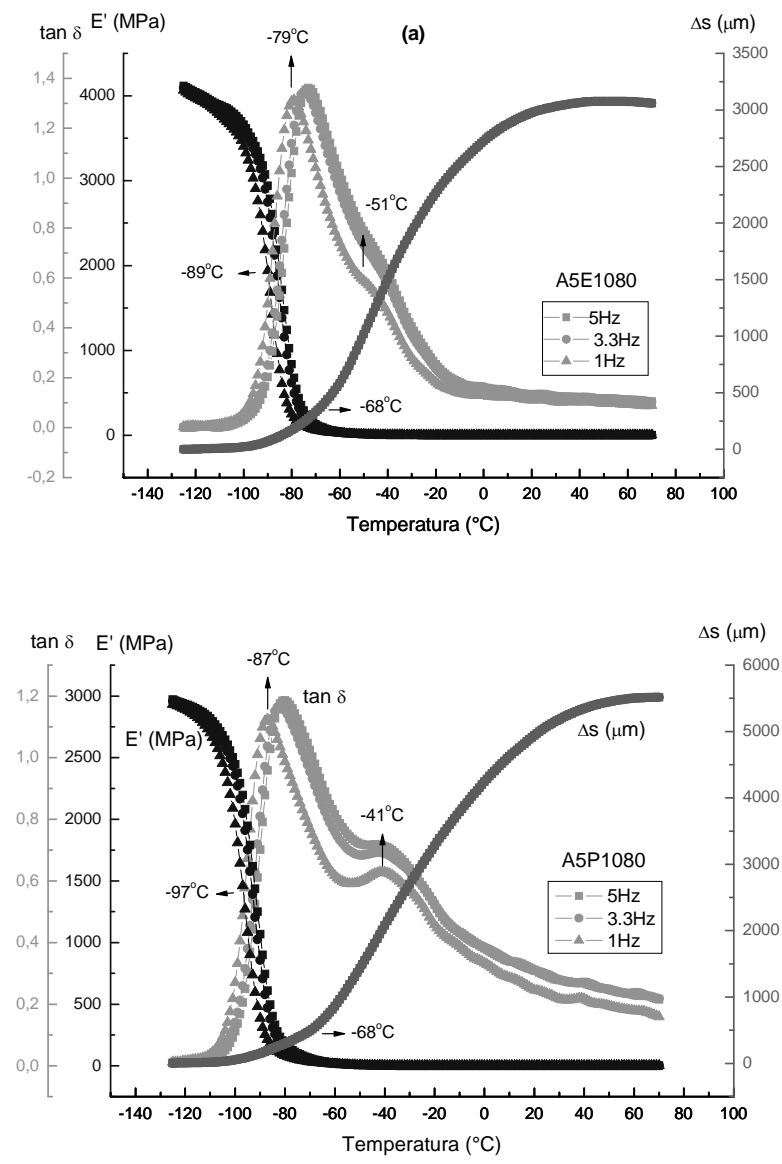

Figura 7: Medidas de Tan $\delta$ módulo elástico E’ e alongamento $\Delta$ s, em função da temperatura, obtidas pela técnica de DMTA, para as amostras (a) A5E1080 e (b) A5P1080, envelhecidas por 1080 horas (6 semanas). 


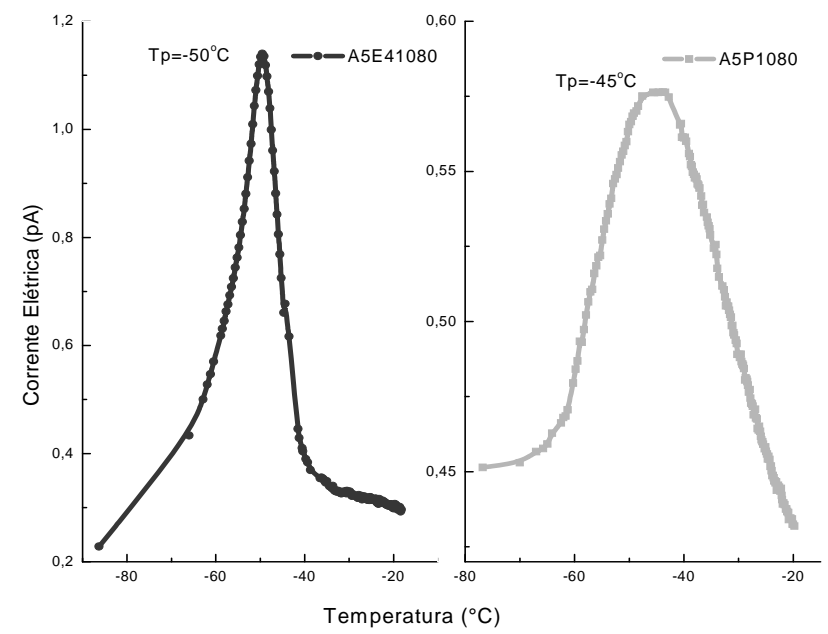

Figura 8: Medidas de TSC para amostras A5E1080 e A5P1080, envelhecidas por 1080 horas (6 semanas).

O caso contrário ao comportamento das temperaturas de relaxação anteriores, ocorre para os resultados mostrados nas Figuras 7 e 8, de DMTA e TSC para 1080h (6 semanas) de envelhecimento térmico. Os valores das temperaturas de relaxações dielétricas e dinâmico-mecânicas tendem a uma ligeira queda. Tal mudança pode ser atribuída ao estágio de degradação no qual é predominante a reação de oxidação nos pontos de cisão das cadeias ou quebra de ligações, resultando em cadeias menores, com maior número de terminações, facilitando, em temperaturas menores, os movimentos moleculares. As diferenças causadas no comportamento dos mecanismos de relaxações dielétricas e dinâmico-mecânicas em função do envelhecimento podem ser melhor visualizadas nas Figuras 9 e 10, que correspondem aos valores de energias de ativação dos processos de relaxação.

As energias de ativação em função do tempo de envelhecimento térmico para os processos dinâmico-mecânicos estudados por DMTA, foram obtidas pela equação de Arrehnius,

$$
f(T)=f_{0} \exp \left(-E_{a} / k T\right)
$$

para as duas composições de BN, com erro experimental de $0,04 \%$ onde $f_{o}$ é o fator pré-exponencial ou a freqüência natural, $E_{a}$ é a energia de ativação, $k$ é a constante de Boltzmann e $T$ é a temperatura em kelvin. A partir do coeficiente angular da reta que melhor ajusta os pontos experimentais no gráfico de $\log f(\mathrm{~Hz})$ versus $1 / \mathrm{T}\left(\mathrm{K}^{-1}\right)$, foram obtidos os valores de energia de ativação $E_{D M T A}$.

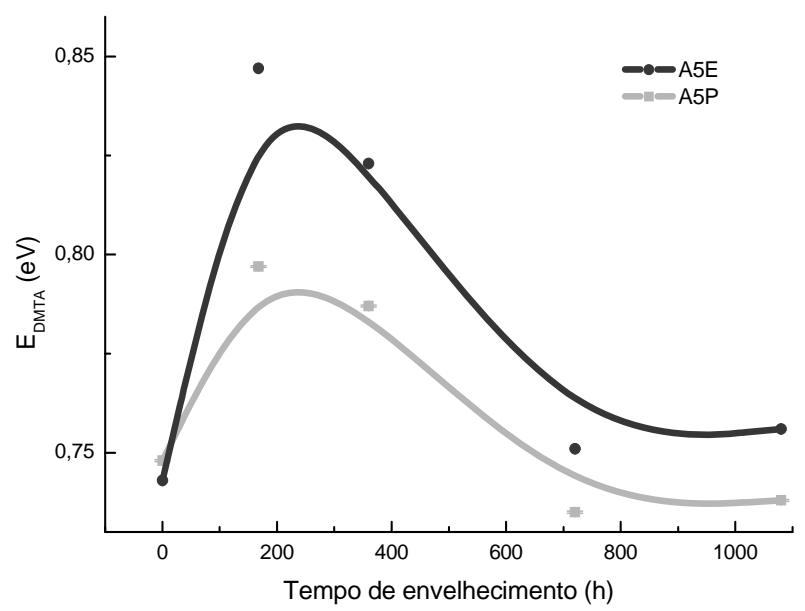

Figura 9: Dependência da energia de ativação $E_{D M T A}$ dos processos dinâmico-mecânicos detectados por DMTA, com o tempo de envelhecimento térmico das amostras A5E e A5P. 
Segundo o modelo de Debye [1] para a relaxação dielétrica, que pode ser explicado adaptando-se a equação de Arrehnius para a corrente de despolarização $i(T)$,

$$
i(T)=i_{0} \exp \left(-E_{a} / k T\right)
$$

foram obtidas as energias de ativação dos mecanismos de relaxação dos dipolos elétricos, detectados por TSC, em função do envelhecimento térmico para as duas formulações, com erro experimental médio de 0,03\%. A partir do coeficiente angular da reta que melhor ajusta os pontos experimentais no gráfico de log $i(A)$ versus $1 / T\left(\mathrm{~K}^{-1}\right)$, foram obtidos os valores de energia de ativação $E_{T S C}$.

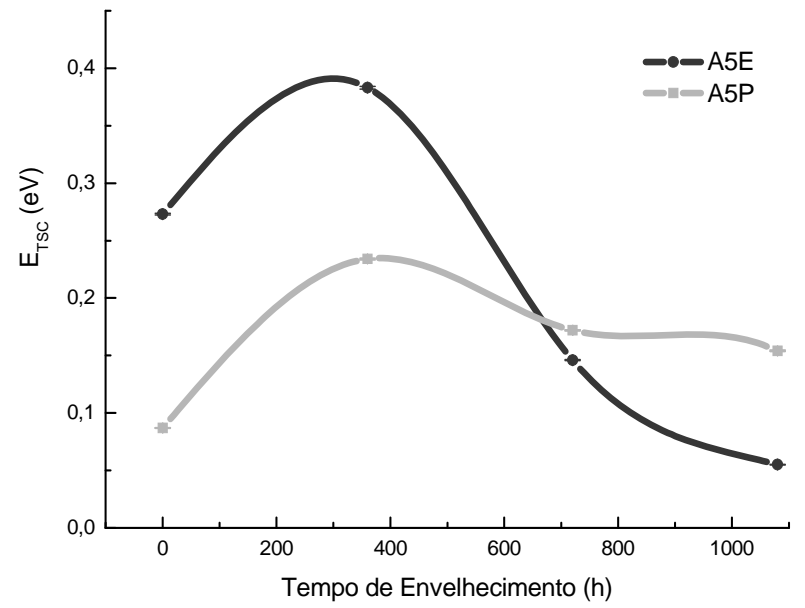

Figura 10: Dependência da energia de ativação $E_{T S C}$ dos mecanismos de TSC com o tempo de envelhecimento térmico das amostras A5E e A5P.

O deslocamento da temperatura de transição vítrea em função do envelhecimento pode ser atribuído à mudança na energia necessária para que os processos dependentes da temperatura ocorram. Nas Figuras $9 \mathrm{e}$ 10, por meio de uma avaliação qualitativa, é possível verificar tais mudanças, e separar a degradação em dois estágios.

No primeiro estágio, em torno de 360h de envelhecimento térmico, o fato dos valores de energia de ativação aumentarem para ambas as relaxações dinâmico-mecânica e dielétrica, sugere que nesta fase de degradação, a reação de reticulação predomina perante a oxidação, para ambas as formulações. Ainda é possível verificar que para a amostra A5E, os valores de energia de ativação são maiores nesta fase de degradação, possivelmente porque a cura via enxofre provoca uma maior densidade de entrecruzamentos entre as cadeias por ligações $C-S_{x}-C$, com energia de ligação $\leq 285 \mathrm{~kJ} / \mathrm{mol}$ [9], que podem ser polissufidicas (via aceleradores) ou monossulfidicas, o que pode contribuir para um aumento na rigidez do polímero. Já a cura por peróxido de dicumila, para as mesmas condições de envelhecimento, por depender da formação de radicais livres que resultam em ligações C-C, com energia de ligação 351 kJ/mol [్], pode resultar numa menor densidade de entrecruzamentos oferecendo mais mobilidade às cadeias [9]. O segundo estágio de degradação é caracterizado pela queda nos valores de energia de ativação tanto para DMTA quanto TSC. Tal queda pode ser atribuída à oxidação das cadeias, resultando numa estrutura menos ligada, mais vulnerável a movimentos moleculares, de ramificações, terminações e segmentos de cadeias. Pode ser atribuída ainda uma maior instabilidade térmica para a formulação de borracha curada com enxofre, por se observar uma maior amplitude de variação nas energias de ativação entre os dois estágios de envelhecimento.

Os valores de energia de ativação determinados pelas duas técnicas abordadas não são iguais, o que é atribuído à diferença nas grandezas medidas, condições e variabilidade de medidas. Porém na tendência qualitativa das mudanças nas propriedades dielétricas e mecânicas fica notável uma relação entre estas propriedades. 


\section{CONCLUSÕES}

A degradação da borracha natural está relacionada em princípio à cisão molecular, que resulta em cadeias menores (oxidação) e num maior número de terminações de cadeias, e/ou reticulação, gerando uma estrutura fortemente ligada (reticulação). Sendo que, dependendo do estágio de degradação, uma das reações pode ser dominante.

Quando se tem uma estrutura quimicamente mais reticulada com o envelhecimento, o que acontece com mais intensidade para A5E, os mecanismos de relaxação mecânica como terminações de cadeias, ramificações, grupos laterais ou segmentos de cadeias e relaxação dielétrica precisam de mais energia para serem ativados, ou seja, a energia necessária para a reorientação dos dipolos ao equilíbrio aumenta. Quando a reação de oxidação predomina, considera-se que, logo após, a estrutura tenha mais liberdade para movimentos de segmentos, ramificações (como ésteres que são grupos altamente polares) e terminações de cadeias poliméricas, possibilitando assim um rearranjo de dipolos.

A densidade de reticulação das cadeias está relacionada com as propriedades mecânicas e dielétricas da borracha natural. Possivelmente para as amostras estudadas, a cura via enxofre provoca uma maior densidade de entrecruzamentos entre as cadeias por ligações $C-S_{x}-C$, que contribui para um aumento na rigidez do polímero. Já a cura por peróxido de dicumila resulta, para as mesmas condições de envelhecimento, por depender da formação de radicais livres que resultam em ligações C-C, numa menor densidade de entrecruzamentos oferecendo mais mobilidade às cadeias, porém oferecendo mais estabilidade térmica ao polímero.

\section{AGRADECIMENTOS}

Ao Instituto de Tecnologia para o Desenvolvimento - LACTEC, à Universidade Federal do Paraná - UFPR, e à Rio Grandense Energia - RGE.

\section{BIBLIOGRAFIA}

[1] ASTM D 120-95: Standard specification for rubber insulating gloves, Philadelphia, ASTM - American Society for Testing and Material, 1995.

[2] ASTM D 1051: Standard specification for rubber insulating sleeves, Philadelphia, ASTM - American Society for Testing and Material, 1995.

[3] ASTM D 178: Standard Specification for Rubber Insulating Matting. Philadelphia, ASTM - American Society for Testing and Material, 1995.

[4] SUH, K.S., DAMON, D., “What is TSC?”, IEEE Electrical Insulation Magazine, v. 8, n. 6, pp. 13-20, December 1992.

[5] BUCCI, C., FIESCHI, R., "Ionic thermoconductivity method for the investigation of polarization in insulators”, Physical Review Letters, v.12, pp. 16-19, January 1964.

[6] BAYARD, J., DARGENT, E., CABOT, C., LEBAUDY, P., GRENET, J., “Thermally stimulated depolarization current measurements - a new relationship to link the dependence of the relaxation time with temperature”, Journal of Thermal Analysis , v. 51, pp. 879-888, September 1998.

[7] HINO, T., “Thermally stimulated characteristics in solid dielectrics”, IEEE Transactions on Electrical Insulation, v. 15, n. 3, pp. 301- 311, June 1980.

[8] TANAKA, Y., "Structural characterization of natural polyisoprenes: solve the mystery of natural rubber based on structural study”, Rubber Chemistry Technology, v. 74, n. 3, pp. 335 - 375, July 2001.

[9] AKCELRUD, L. Fundamentos da Ciência dos Polímeros, Barueri, Manole, 2007.

[10] MARINHO, J.R.D., Macromoléculas e Polímeros, Barueri, Manole, 2005.

[11] LUCAS, E.F., SOARES, B.G., MONTEIRO, E., Caracterização de polímeros: determinação do peso molecular e análise térmica, Rio de Janeiro, E-papers, 2001. 
[12] LEYVA, M.E., BARRA, G.O., SOARES, B.G., KHASTGIR, D., “Mistura Pani.DBSA/SBS obtida por polimerização “In Situ”: propriedades elétrica, dielétrica e dinâmico-mecânica”, Polímeros: Ciência e Tecnologia, v. 12, n. 3, pp. 197-205, Outubro 2002.

[13] SANCHEZ, E.M.S., FERREIRA, M.M.C., FELISBERTI, M.I. “Avaliação da degradação térmica e fotooxidativa do ABS automotivo”, Polímeros: Ciência e Tecnologia, v. 12, n. 9, pp. 116, Outubro 1999.

[14] ESCÓCIO, V.A., MARTINS A.F., VISCONTE L.L.Y., NUNES R.C.R., "Efeito do envelhecimento nas propriedades mecânicas e dinâmico-mecânicas de composições de borracha natural com mica”, Polímeros: Ciência e Tecnologia, v. 14, n. 1, pp. 13-16, Março 2004.

[15] MUNARO, M., Desenvolvimento de Blendas de Polietileno com Desempenho Aperfeiçoado para Utilização no Setor Elétrico, Tese de D.Sc., PIPE/UFPR, Curitiba, PR, Brasil, 2007.

[16] KOWALSKI, E.L., Estudo da Borracha Natural Por Meio de Técnicas de Caracterização de Dielétricos, Tese de D.Sc., PIPE/UFPR, Curitiba, PR, Brasil, 2006.

[17] OLIVEIRA, F.A., ALVES, N., GIACOMETTI, J.A., CONSTANTINO, C. J. L., MATTOSO, L.H.C., BALAN, A.M.O.A., JOB, A.E., "Study of the thermomechanical and electrical properties of conducting composites containing natural rubber and carbon black", Journal of Applied Polymers Science, v. 106, pp. 1001-1006, July 2007.

[18] DEBYE, P.J.W., Polar Molecules, New York, Dover Publications Inc., 1929. 